\title{
Compound heterozygous IFT140 variants in two Polish families with Sensenbrenner syndrome and early onset end-stage renal disease
}

Joanna Walczak-Sztulpa ${ }^{1 *} \mathbb{D}$, Renata Posmyk ${ }^{2+}$, Ewelina M. Bukowska-Olech ${ }^{1 \dagger}$, Anna Wawrocka ${ }^{1}$, Aleksander Jamsheer', Machteld M. Oud ${ }^{3,4}$, Miriam Schmidts ${ }^{3,5}$, Heleen H. Arts ${ }^{6,7}$, Anna Latos-Bielenska ${ }^{1}$ and Anna Wasilewska ${ }^{8}$

\begin{abstract}
Background: Sensenbrenner syndrome, which is also known as cranioectodermal dysplasia (CED), is a rare, autosomal recessive ciliary chondrodysplasia characterized by a variety of clinical features including a distinctive craniofacial appearance as well as skeletal, ectodermal, liver and renal anomalies. Progressive renal disease can be life-threatening in this condition. CED is a genetically heterogeneous disorder. Currently, variants in any of six genes (IFT122, WDR35, IFT140, IFT43, IFT52 and WDR19) have been associated with this syndrome. All of these genes encode proteins essential for intraflagellar transport (IFT) a process that is required for cilium assembly, maintenance and function. Intra- and interfamilial clinical variability has been reported in CED, which is consistent with CED's genetic heterogeneity and is indicative of genetic background effects.

Results: Two male CED patients from two unrelated Polish families were included in this study. Clinical assessment revealed distinctive clinical features of Sensenbrenner syndrome, such as dolichocephaly, shortening of long bones and early onset renal failure. Ectodermal anomalies also included thin hair, short and thin nails, and small teeth in both patients. Next generation sequencing (NGS) techniques were performed in order to determine the underlying genetic cause of the disorder using whole exome sequencing (WES) for patient 1 and a custom NGS-based panel for patient 2. Subsequent qPCR and duplex PCR analysis were conducted for both patients. Genetic analyses identified compound heterozygous variants in the IFT140 gene in both affected individuals. Both patients harbored a tandem duplication variant p.Tyr1152_Thr1394dup on one allele. In addition, a novel missense variant, p.(Leu109Pro), and a previously described p.(Gly522Glu) variant were identified in the second allele in patients 1 and 2, respectively. Segregation analysis of the variants was consistent with the expected autosomal recessive disease inheritance pattern. Both patients had severe renal failure requiring kidney transplantation in early childhood.
\end{abstract}

Conclusion: The finding of compound heterozygous IFT140 mutations in two unrelated CED patients provide further evidence that IFT140 gene mutations are associated with this syndrome. Our studies confirm that IFT140 changes in patients with CED are associated with early onset end-stage renal disease. Moreover, this report expands our knowledge of the clinical- and molecular genetics of Sensenbrenner syndrome and it highlights the importance of multidisciplinary approaches in the care of CED patients.

Keywords: Sensenbrenner syndrome, Cranioectodermal dysplasia, IFT140, Ciliopathy, End-stage renal disease

\footnotetext{
*Correspondence: jsztulpa@ump.edu.pl

${ }^{\dagger}$ Renata Posmyk and Ewelina M. Bukowska-Olech contributed equally to this work.

'Department of Medical Genetics, Poznan University of Medical Sciences,

Rokietnicka 8 Street, 60-608 Poznan, Poland

Full list of author information is available at the end of the article
}

(c) The Author(s). 2020 Open Access This article is distributed under the terms of the Creative Commons Attribution 4.0 International License (http://creativecommons.org/licenses/by/4.0/), which permits unrestricted use, distribution, and reproduction in any medium, provided you give appropriate credit to the original author(s) and the source, provide a link to the Creative Commons license, and indicate if changes were made. The Creative Commons Public Domain Dedication waiver (http://creativecommons.org/publicdomain/zero/1.0/) applies to the data made available in this article, unless otherwise stated. 


\section{Background}

Sensenbrenner syndrome is an ultra-rare autosomal recessive disorder that is thought to result from dysfunction of cilia. CED is a genetically heterogeneous disease affecting multiple systems. It is diagnosed based on characteristic clinical features, which include sagittal craniosynostosis, dolichocephaly, facial dysmorphisms such as epicanthal folds, telecanthus, hypertelorism and frontal bossing, growth retardation, shortening of upper and lower limbs, narrow thorax, protuberant abdomen, progressive renal disease and ectodermal abnormalities. Liver and retinal dysfunction have also been reported in CED patients, albeit less frequently $[1,2]$.

To date, more than 60 patients have been reported in literature and mutations in six genes have been associated with Sensenbrenner syndrome: IFT122, WDR35, IFT140, IFT43, IFT52 and WDR19 [3-8]. All of these genes encode proteins that are involved in intraflagellar transport (IFT). This is a bi-directional transport process that occurs in the cilium and plays a crucial role in cilium assembly, maintenance and function. The IFT140 protein is part of the so-called IFT-A complex that primarily regulates retrograde intraflagellar ciliary transport (i.e., transport of cargo proteins from the ciliary tip to its base). The IFT140 gene consists of 31 exons (29 coding exons) and encodes a 1462 amino acid protein that contains five WD repeats and nine tetratricopeptide (TPR) repeats [9-11].

Mutations in IFT122 and WDR35 are the most common cause of CED and explain about $60 \%$ of families with Sensenbrenner syndrome. To date, only two unrelated CED patients with IFT140 variants have been reported in literature [8, 12]. IFT140 mutations thus constitute a relatively uncommon cause of CED. However, clinical phenotypes associated with dysfunction of IFT140 are not limited to CED. In fact, pathogenic variation in IFT140 has also been reported in patients with Mainzer-Saldino Syndrome (MSS), Jeune Syndrome (JATD), Opitz trigonocephaly C syndrome (OTCS), and isolated retinal dystrophy [13, 14]. CED, MSS and JATD are phenotypically and genetically related disorders, and are collectively referred to as short-rib thoracic dysplasia syndromes [15]. The identification of variants in IFT140 in patients with various, overlapping phenotypic features is in line with the general presumption that ciliopathies represent a spectrum of disorders with marked phenotypic and genotypic overlap among distinctly-classified conditions.

\section{Clinical examination}

In our study two male patients from two unrelated, nonconsanguineous Polish families were diagnosed with Sensenbrenner syndrome.

\section{Patient 1}

A 3.5-years-old boy was referred to the Genetic Counseling Unit due to renal failure, skeletal abnormalities and ocular problems. The patient was born to young (20-year-old mother and 33-year-old father), unrelated parents from a first, unremarkable pregnancy, by conventional vaginal delivery in the 39th week of gestation. The birth weight was $3700 \mathrm{~g}$ (50th -75 th centile), length $57 \mathrm{~cm}$ (97th centile), head circumference $36 \mathrm{~cm}$ (75th97th centile), and thorax circumference $33 \mathrm{~cm}$ (25-50th centile). The Apgar score was 9 at $1 \mathrm{~min}$. The family history was unremarkable. Although the family doctor recorded proteinuria, hematuria and glycosuria in the early neonatal period, no further evaluation was conducted. At the age of 12 months the child was treated for a urinary tract infection. At three years of age, he developed bronchopneumonia and was hospitalized in a district hospital, where elevated creatinine levels were detected. The child was referred to the Pediatric Nephrology Department for further diagnosis. Routine laboratory investigations showed constant proteinuria, glycosuria, hematuria, eGFR (Schwartz)- 30,56 ml/ $\mathrm{min} / 1.73 \mathrm{~m} 2$, elevated serum levels of creatinine, uric acid and urea, total cholesterol, and triglyceride were determined, whereas complete blood count, blood glucose, albumin, thyroid and liver function tests were normal. Ultrasonography of kidneys showed increased renal cortex echogenicity and decreased corticomedullary differentiation.

Distinctive dysmorphic features were observed during the first assessment by a clinical geneticist at the age of 3.5 years. The features included dolichocephaly, high forehead, thin hair, full cheeks, low set prominent ears, long philtrum, microretrognathia, rhizomelic shortening of upper and lower limbs, brachydactyly of toes and fingers, narrow chest and pectus excavatum. These features, in combination with progressive renal failure, were indicative of a ciliopathy (Fig. 1 a-d and Table 1). Ophthalmological examination confirmed strabismus, nystagmus and high hyperopia. Cytogenetic analysis (conventional GTG banding) revealed a normal male karyotype $(46, \mathrm{XY})$. The patient was seen in the genetics clinic every 6 months. Renal function rapidly declined in the following 12 months. At the age of 4.5 years the patient had developed end-stage renal failure and peritoneal dialysis was ordered. At the age of 6.5 years, his weight was $19.3 \mathrm{~kg}$ (25th50th centile), his height was $102 \mathrm{~cm} \mathrm{(<3rd} \mathrm{centile),}$ and his BMI was $18,55 \mathrm{~kg} / \mathrm{m} 2$ (97th centile). His morphological phenotype markedly differed from the previous clinical assessments during the last examination at the age of 9 years (Fig. $1 \mathrm{e}-\mathrm{h}$ ). Short stature, obesity, short fingers and toes, and shortening of the long bones were more prominent. Dolichocephaly was less 


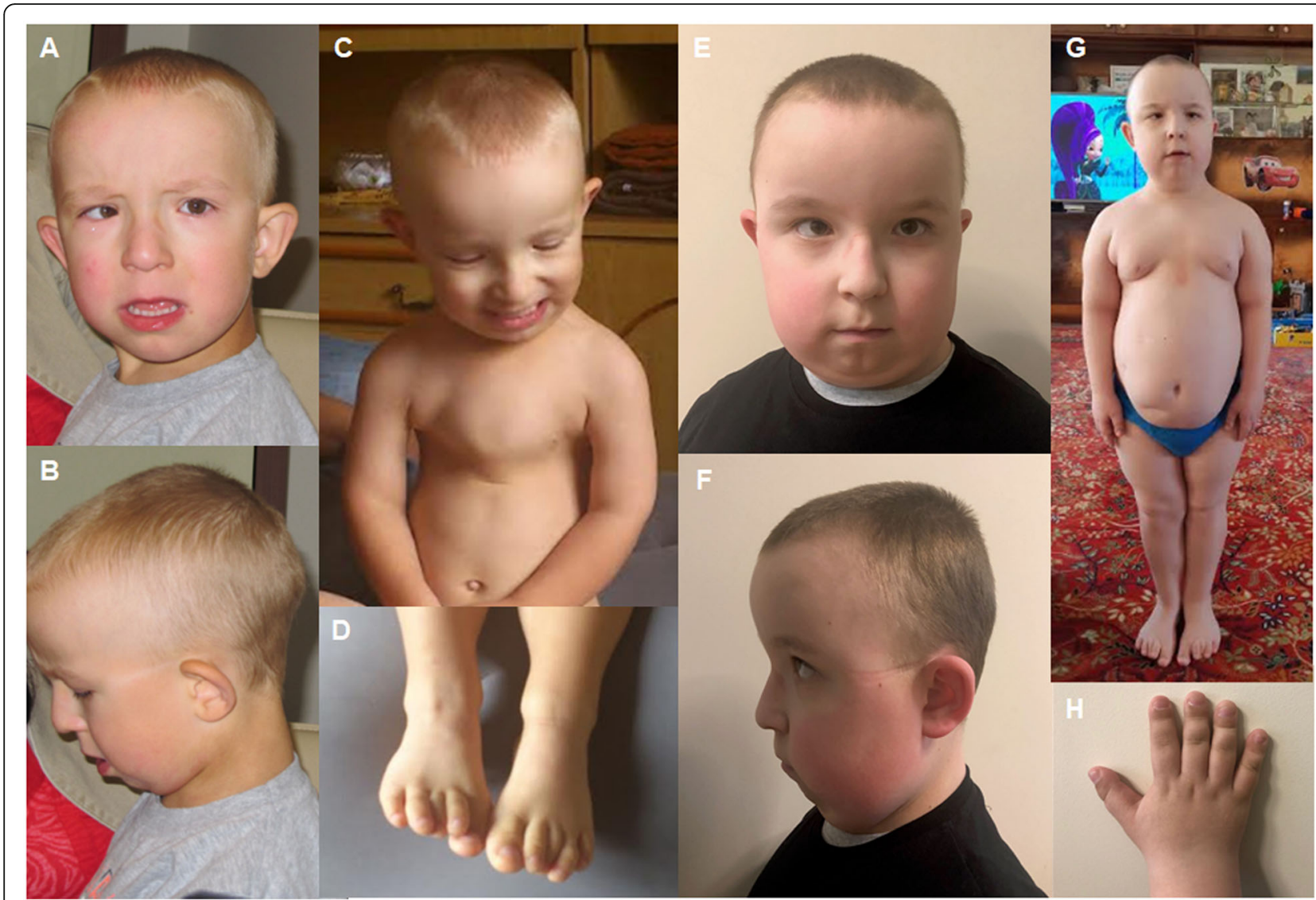

Fig. 1 Dysmorphic features and changing phenotype of patient 1 at the age of 3 y $7 \mathrm{~m}$ (a-d) and 9 y $3 \mathrm{~m}$ (e-h) respectively. (a, e) Characteristic facial features. Facial features are dolichocephaly, high prominent forehead, thin sparse hair, full cheeks, strabismus, broad prominent nasal bridge, flat philtrum columns, narrow mucus upper lip, everted lower lip, open mouth, low-set prominent ears, microretrognathia. (b, f) A facial lateral view showing low set ears and sparse hair. (c) Narrow chest, pectus excavatum. (d) Brachydactyly and overlap of toes. (g) Whole body image showing proximal limb shortening, a short broad neck, a narrow thorax and obesity. (h) Brachydactyly of the right hand

evident. Psychomotor development was normal. To date, he attends a normal primary school; he is a very intelligent and positively oriented boy.

\section{Patient 2}

A 14-month-old male patient was referred to our clinic with suspicion of chondrodysplasia. The patient was born at 40 weeks of gestation, after a first, uneventful pregnancy. He was delivered by Cesarean section. Anthropometric measurements were in the normal range: his birth weight was $3500 \mathrm{~g}$ (50th-75th centile), his body length was $58 \mathrm{~cm}$ (97th centile), his head circumference was 34 cm (25th-50th centile), his thorax circumference was 33 $\mathrm{cm}$ (25th-50th centile) and he had an Apgar score of 10 . His parents, a 28-year-old mother and a 33-year-old father, are healthy and have a non-consanguineous relationship. The family history was unremarkable. The neonatal period was complicated by recurrent respiratory infections, mild hypotonia, and atopic skin. The first renal problems, proteinuria and hematuria, were observed in the 7th month. The boy was hospitalized at 8th months and a full diagnostic assessment was performed. The ultrasound revealed bilateral enlarged kidneys (left $61 \mathrm{~mm}$ and right $63 \mathrm{~mm}$ ) with edema and poor cortico-medullary differentiation. Renal biopsy showed a chronic injury of the parenchyma. Due to progressive renal failure and an advanced stage of renal insufficiency, peritoneal dialysis was initiated. Renal transplantation has been suggested for this patient. Anomalies of other internal organs were not identified. Hyperopia and nystagmus were noted during ophthalmological examination.

The patient was first seen by a clinical geneticist at the age of 14 months. The geneticist noted that the patient had a short stature with rhizomelic shortening in upper and lower extremities. Distinctive dysmorphic features indicative of Sensenbrenner syndrome were found. These included dolichocephaly, high prominent forehead, "senile-like" face, very thin sparse hair, full cheeks, thin upper and lower lip, low-set protruding ears, pointed chin and small teeth (Fig. 2a-f and Table 1). 
Table 1 Clinical features of CED patients carrying compound heterozugous variants in IFT140

\begin{tabular}{|c|c|c|}
\hline Patient & 1 & 2 \\
\hline Variant (DNA) & c.326 T > C + c.3454-488_4182+2588dup & c.1565G > A + + c.3454-488_4182+2588dup \\
\hline Variant (protein) & p.(Leu109Pro) + p.Tyr1152_Thr1394dup & p.(Gly522Glu) + p.Tyr1152_Thr1394dup \\
\hline Age at examination & 3 years 6 months & 14 months \\
\hline Sex & male & male \\
\hline Dolichocephaly & + & + \\
\hline Craniosynostosis & + & - \\
\hline Frontal bossing & + & + \\
\hline Full cheeks & + & + \\
\hline Broad nasal bridge & + & + \\
\hline Long philtrum & + & + \\
\hline Flat philtrum columns & + & - \\
\hline Narrow mucus upper lip & + & + \\
\hline Everted lower lip & + & - \\
\hline Low set ears & + & + \\
\hline Protruding ears & + & + \\
\hline Narrow chest & + & + \\
\hline Pectus excavatum & + & - \\
\hline Short ribs & + & + \\
\hline Wide ribs & + & + \\
\hline Rhizomelic limb shortening & + & + \\
\hline Short humeri & + & + \\
\hline Short fibulae & + & + \\
\hline Flattened epiphyses & + & + \\
\hline Brachydactyly of fingers and toes & + & + \\
\hline Overlapping toes & + & - \\
\hline Joint laxity & + & + \\
\hline Fine, sparse hair & + & + \\
\hline Small teeth & + & + \\
\hline Thin nails & + & + \\
\hline Short nails & + & + \\
\hline Protuberant abdomen & + & + \\
\hline Progressive renal failure & + & + \\
\hline Tubulointerstitial nephritis & + & + \\
\hline Ophthalmological problems & Hyperopia, strabismus, nystagmus & Hyperopia \\
\hline Recurrent respiratory infections & + & + \\
\hline Intelligence & Normal & Normal \\
\hline Motor development & Normal & Delayed (walking at 22 months) \\
\hline
\end{tabular}

Developmental milestones were delayed: the patient was able to sit independently at 12 months, but did not crawl. He started walking at 22 months and began to say a few simple words.

Patient 1 and patient 2 presented with very similar phenotypes and a clinical diagnosis of Sensenbrenner syndrome was made for both patients (Table 1).

\section{Results}

Genetic analysis

Next generation sequencing was performed for both patients in order to determine the molecular cause of CED. A whole-exome was sequenced in patient 1 and a custom panel of 61 genes and 11 SNPs was sequenced for patient 2. Genetic analyses were 


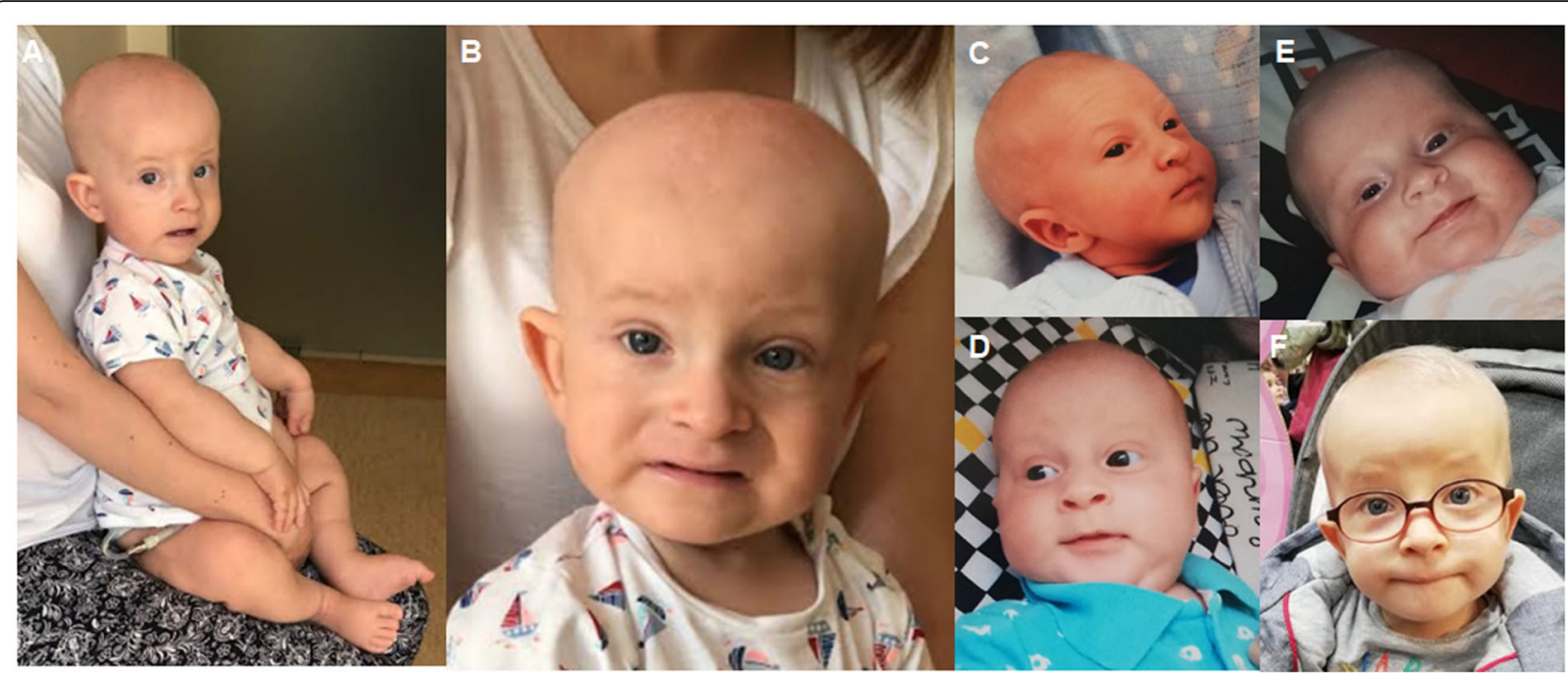

Fig. 2 Dysmorphic features and changing phenotype of patient 2. At the age of 14 months (a, b). Facial features including dolichocephaly, high prominent forehead, "senile-like" face, very thin sparse hair, full cheeks, thin upper and lower lip, low-set protruding ears, pointed chin, small teeth. (c) Newborn period, (d) 2-month-old, (e) 4-month-old, (f) 23-month-old

complemented by qPCR and duplex PCR analysis for both patients.

\section{Patient 1}

Whole exome sequencing (WES) revealed a heterozygous missense variant c.326 T $>C$; p.(Leu109Pro) in IFT140. Subsequent qPCR and duplex PCR analysis in a combination with Sanger sequencing showed a heterozygous tandem duplication c.3454-488_4182 + 2588dup; p.Tyr1152_Thr1394dup on the other allele. The variants were inherited from the patient's unaffected father and mother, respectively. The tandem duplication was not identified through WES analysis.

The missense substitution p.(Leu109Pro) is a novel variant, which has not previously been reported in the HGMD database. The variant was not reported in the 1000 Genomes, the NHLBI Exome Variant Server (EVS), the Exome Aggregation Consortium (ExAC) or the gno$\mathrm{mAD}$ database (25.07.2019). This change was predicted to be pathogenic by MutationTaster, PolyPhen and SIFT software. We classified the p.(Leu109Pro) variant as likely pathogenic based on variant classification guidelines from the American College of Medical Genetics and Genomics and the Association for Molecular Pathology [16].

\section{Patient 2}

Sequencing of a custom gene panel by NGS revealed a heterozygous missense variant c.1565G > A; p.(Gly522Glu) in IFT140 in the patient. Subsequent qPCR and duplex PCR analysis in combination with Sanger sequencing identified the same heterozygous tandem duplication that was observed in patient 1 . The variants were inherited from the patient's unaffected mother and father, respectively. This tandem duplication was not detected by NGS analysis.

The p.(Gly522Glu) variant was listed in the Exome Aggregation Consortium database (ExAC) and has been reported in as a heterozygous variant in 17 out of 121,256 analyzed alleles, while this variant has been reported as a heterozygous variant in 39 out of 277,186 alleles in the gnomAD database. According to the ExAC and gno$\mathrm{mAD}$ databases $(25.07 .2019)$, the variant frequency is $0.01402 \%$ and $0.01407 \%$, respectively. This change was not listed in the Exome Variant Server (EVS) database. In silico tools SIFT, PolyPhen, MutationTaster predict that this change has a deleterious effect on IFT140 protein. The variants was classified as likely pathogenic by ACMG guidelines [16]. In both families, segregation analysis of the identified variants was consistent with an autosomal recessive inheritance pattern of the disease (Fig. 3a, b), which provides further evidence for causality.

\section{Breakpoint analysis}

Sanger sequencing of the breakpoints confirmed the presence of a tandem duplication spanning exons 27 to 30 of the IFT140 gene in both patients. Breakpoint sequencing results are shown in Fig. 3c.

\section{Discussion}

We identified compound heterozygous causal variants in IFT140 in two unrelated Polish CED patients with early onset renal disease. 

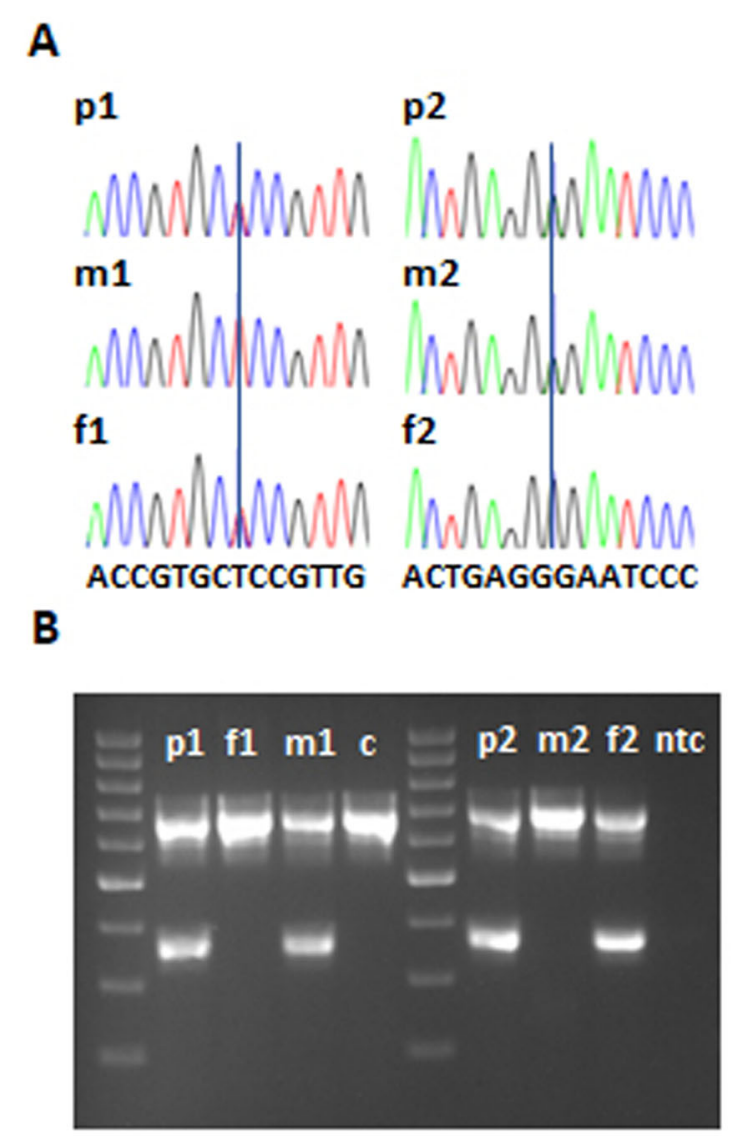

C

Intron $\mathbf{3 0}$

Intron 26

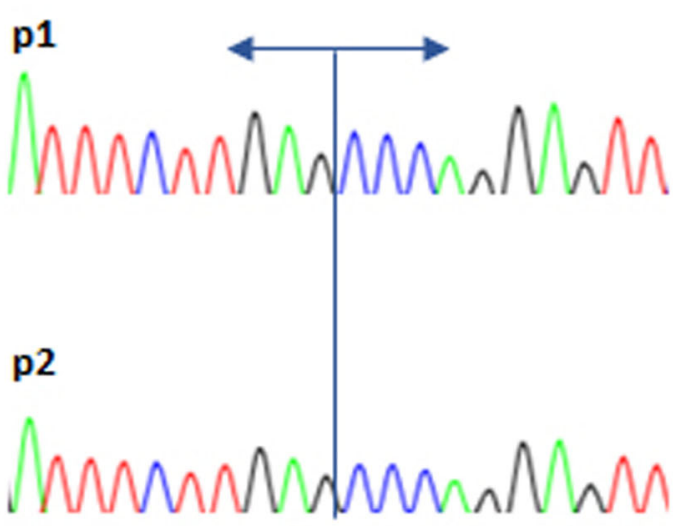

ATTTCTTGAGCCCAGGAGTT
Fig. 3 IFT140 variants segregated with disease in both families with Sensenbrenner syndrome. Patient 1 has a heterozygous c.326 T > C (p.(Leu109Pro)) variant, which was inherited from the mother (a) and patient 2 has a heterozygous c.1565G > A (p.(Gly522Glu)) change, which was inherited from the father (b). Duplex-PCR revealed the presence of a tandem duplication p.Tyr1152_Thr1394dup in patients 1 and 2. This variant was inherited from the father in patient 1 and from the mother in patient 2, respectively $(\mathbf{c})$. Sanger sequencing traces showing the breakpoints of the tandem duplication p.Tyr1152_Thr1394dup in both CED patients (d)

Mutations in the IFT140 gene are associated with isolated retinal dystrophy, Mainzer-Saldino (MSS) Syndrome, Jeune Syndrome (JATD) and Opitz trigonocephaly C syndrome (OTCS) $[13,14]$. JATD, MSS and CED are clinically overlapping disorders. WDR35 and IFT122 genes are most commonly mutated in Sensenbrenner syndrome, and variants in the IFT140 gene are a rare cause of CED. Genetic analyses conducted in the present study revealed compound heterozygous variants in the IFT140 gene in both unrelated CED patients. Both patients have a heterozygous tandem duplication p.Tyr1152_Thr1394dup on one allele in combination with a heterozygous missense variant on the second allele, p.(Leu109Pro), in patient 1, and a p.(Gly522Glu) variant in patient 2 . Segregation analysis was performed in both families and the results were consistent with an autosomal recessive inheritance mode. Our results provide further support that the clinical spectrum associated with IFT140 variants includes CED, which is important as current knowledge on the association of IFT140 with CED is only marginal $[8,12]$.

The p.Tyr1152_Thr1394dup tandem duplication in IFT40 is a known disease-associated variant that has recently been reported in eight families [12]. Seven patients of six of these families were diagnosed with Mainzer-Saldino syndrome, one patient had a phenotype reminiscent of Jeune syndrome and one patient had features of Sensenbrenner syndrome. This duplication is predicted to be in-frame and is thought to result in the addition of 243 amino acids within the tetratricopeptide repeat (TPR). RNA analysis showed that the duplicated exons are transcribed. However, western blotting did not detect the altered protein based on electrophoretic migration [12].

The missense variant p.(Leu109Pro) detected in patient 1 is a novel variant located in the WD40 functional domain of the IFT140 protein. In silico analyses based on SIFT, PolyPhen- 2 and MutationTaster 2 showed that this change is predicted to disrupt the IFT140 protein and is likely pathogenic.

The p.(Gly522Glu) change has been previously reported in a patient with Mainzer-Saldino syndrome and is predicted to have a deleterious effect on the IFT140 protein [9]. The location of the variants identified in both CED families is shown 
in a diagram of the IFT140 gene and encoded protein in Fig. 4.

Both patients described here displayed early onset of renal disease. Patient 1 had a kidney transplantation at the age of 6 years and patient 2 started peritoneal dialysis at the age of 8 months with a recommendation for renal replacement due to an advanced stage of renal insufficiency. To date, only two CED patients harboring IFT140 mutations have been described in literature: a male patient, reported by Bayat et al., who received a kidney transplant at the age of 4 years [8] and a female patient, described by Geoffroy et al., who developed endstage renal failure, requiring dialysis at the age of 3.8 years and a renal transplant thereafter [12]. We conclude that progressive and end-stage renal disease may have an early onset in CED patients with IFT140 variants.

Currently, there is no therapy available to significantly delay or prevent end-stage renal disease for patients diagnosed with autosomal recessive renal ciliopathies. Treatment options are limited to dialysis and transplantation. Typically, there is a 5-10 year interval between diagnosis of renal insufficiency and renal replacement, which provides a window of opportunity for therapeutic treatment of these patients. It is, however, challenging to develop therapies as generation of animal models is both laborious and costly given the enormous genetic heterogeneity that characterizes the renal ciliopathies. The low frequency of this group of disorders in the population is another complicating factor that impacts costeffectiveness of therapy development based on animal models.

An alternate and more cost-effective approach is to assess potential therapeutic effects of drugs in the patient's own cells. Induced pluripotent stem cells (iPSCs) technology may be a powerful tool to facilitate personalized drug assessment as iPSCs can be derived from fluids and tissues that are easy to obtain (blood, fibroblasts and urine) and can subsequently be differentiated into cells of a relevant tissue. Thus, a personalized approach for drug screening and gene-based therapy may be the future direction for individuals diagnosed with Sensenbrenner syndrome $[17,18]$.

The use of iPSCs is not only promising for personalized therapeutic assessments, it can also be helpful in making a diagnosis. For example, Forbes et al. recently described a patient with Mainzer-Saldino syndrome with compound heterozygous variants in IFT140 for whom the iPSC technology was used to facilitate diagnosis. In this study renal organoids representing the developing nephron were created from patient-derived iPS cells (derived from skin-fibroblasts) in order to validate the clinical suspicion of a renal ciliopathy. These patient-derived organoids showed shortened and club-shaped primary cilia. This result is consistent with IFT140 dysfunction as loss of IFT140 function has been associated with shortened cilia and an accumulation of proteins at the ciliary tip [19]. By using proband-derived cell lines followed by rescue with CRISPR editing genetic differences between test and control lines can be reduced; Forbes et al. showed that CRISPR-Cas9 gene correction of IFT140 could rescue the ciliary phenotype seen in their patient-derived organoids, thereby providing additional evidence that abnormal IFT140 caused the ciliary defects in this patient [11].

It has been suggested that gender plays a role in the severity of kidney dysfunction and that in males renal disease progresses more rapidly than in females, which implies that that sex hormones may be essential for direct or indirect modulation of the progression rate of renal disease [20]. It has been shown that kidney dysfunction is more often present in male CED patients with IFT122 and WDR35 than in female patients [3, 21, 22]. So far only one male and one female patient with IFT140 variants were described in the literature and both developed early onset of renal disease and received a kidney transplant $[8,12]$. Further clinical data is needed in order to confirm this possible gender effect in individuals with Sensenbrenner syndrome.

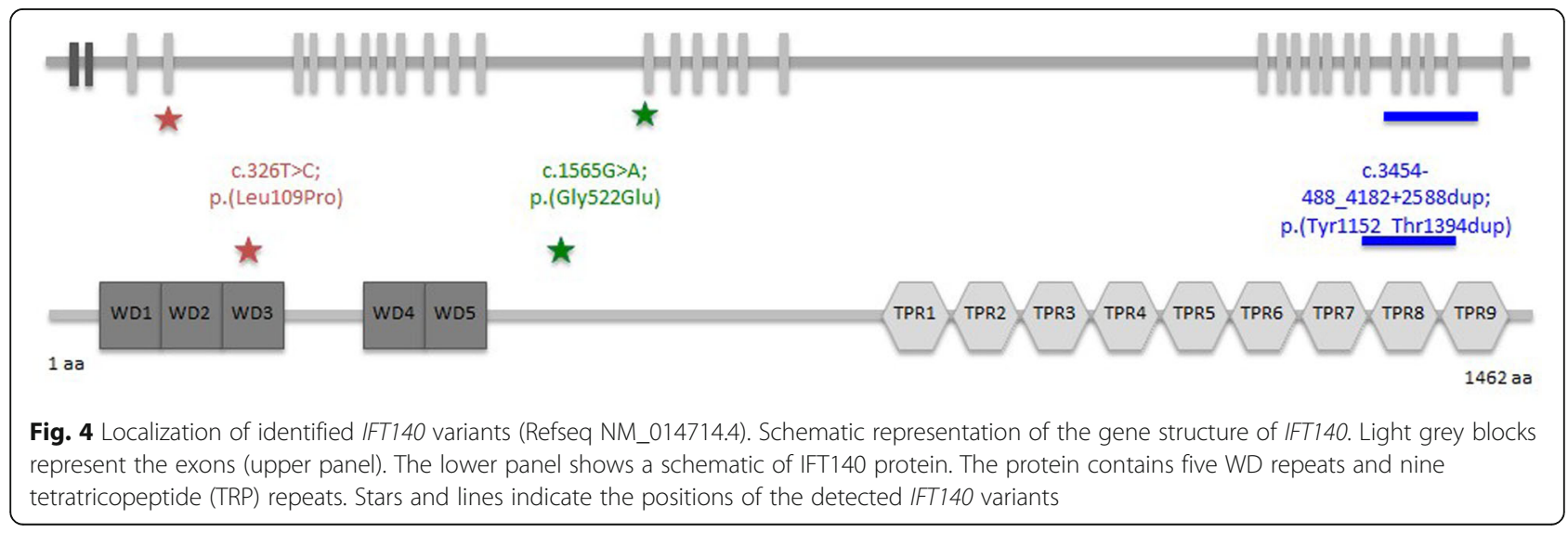


Signs of retinal disease have been reported in patients with variants in IFT140 [12, 13]. Ophthalmological assessments of both patients presented in this study revealed nystagmus and hyperopia. In addition, patient 2 presented with strabismus. Similar ocular findings have been reported in a patient with features of MSS/CED with IFT140 variants described by Geoffroy et al., while the patient described by Bayat et al. was diagnosed with retinal dystrophy showing that ocular defects are probably a frequent feature in CED patients with IFT140 mutations $[8,12]$.

\section{Conclusions}

In our study, we identified compound heterozygous IFT140 variants in two CED patients using NGS combined with qPCR, duplex PCR and Sanger sequencing analysis. Without the use of qPCR and duplex PCR, one of the mutations p.Tyr1152_Thr1394dup would have been missed in each of our patients and they would have lacked a molecular diagnosis. Targeted analysis with these or comparable methods in unexplained CED patients who have a single heterozygous pathogenic variant in IFT140 should therefore be considered. We found that individuals affected by Sensenbrenner syndrome and IFT140 defects developed early onset end-stage renal disease. This finding may ultimately contribute to a better understanding of genotype-phenotype correlations in CED. This is important as not all CED patients develop rapidly progressive renal disease in early childhood and the molecular background of the variable kidney dysfunction seen in CED remains poorly understood. Finally, inter- and intrafamilial clinical variability further complicate the provision of an accurate prognosis for patients and their families [23]. Early monitoring of renal function is therefore warranted in patients with cranioectodermal dysplasia.

\section{Methods \\ Molecular analysis}

EDTA blood samples from the affected individuals and their parents were obtained for genetic analyses. DNA was extracted from peripheral leukocytes using a standard protocol.

\section{Whole-exome sequencing}

Whole-exome sequencing was conducted on genomic DNA from patient 1. Exome enrichment was performed using a SureSelect Human All Exon kit v5 50Mb kit (Agilent, Santa Clara, USA) followed by sequencing on a HiSeq4000 system (Illumina, San Diego, CA, USA). Read mapping was performed using the Burrows-Wheeler Alignment Tool (BWA) and variants were called using the Genome Analysis Toolkit (GATK) haplotypecaller. Variant annotation was performed using the in-house annotation pipeline [24]. To prioritize variants in the sequencing data, variants that were non-genic, intronic (except for canonical spice sites) or synonymous were excluded. Variants that were present in the dbSNPv135 at a frequency of $>1 \%$ or $>1 \%$ in the in-house variants database were also excluded. A quality filter excluded variants with $<5$ reads or $<20 \%$ variant reads.

\section{Targeted next-generation sequencing}

A custom NGS gene panel encompassing 61 genes and 11 SNPs $(225.709 \mathrm{~kb})$ associated with craniofacial malformations was sequenced in patient 2. An additional file contains a list of genes and SNPs included in the NGS gene panel (see Additional file 1). NGS libraries were prepared using a SureSelect-based enrichment approach (Agilent Technologies) and these were subsequently sequenced on the Ion Torrent S5 platform. Reads were demultiplexed and aligned to the GRCh37 human reference genome using TorrentBrowser 5.0.4 software. The resulting BAM files were further processed using IonReporter 5.2. Estimates of coverage for individual genes were obtained by using bedtools 2.27.1 with a BED file defining coding parts of canonical transcripts. Variant quality control was based on four metric parameters; selection of variants with read depth $\geq 20$, PHRED scores $>30$ and variant frequencies of $>15 \%$, and avoidance of strand-specific sequencing errors by excluding variants that occur with variant frequencies differing $>80 \%$ on opposite strands. In silico predictions based on SIFT, PolyPhen and PhyloP (46-way) were used to categorize the functional relevance of genetic variants and this information was retrieved from the IonReporter result files. In addition, CADD scores were evaluated, and bioinformatic prediction programs such as MutationTaster and SnpEff were used for variant classification. Population-specific allele frequencies were derived from Ensemble/VEP and gnomAD databases.

\section{Sanger sequencing}

The presence of the through NGS identified missense variants was confirmed by Sanger sequencing. Primers were designed using Primer3. An additional file contains a list of primer sequences used for PCR and Sanger sequencing (see Additional file 2). PCR reactions in a total volume of $10 \mu \mathrm{l}$ contained $1 \mu \mathrm{l}$ of genomic DNA (100 ng/ $\mu \mathrm{l}), 5 \mu \mathrm{l}$ of 10x FailSafe Premix J buffer (Epicentre Biotechnologies), $0.5 \mu \mathrm{l}$ of forward and reverse primer each $(10 \mu \mathrm{mol} / \mathrm{l}), 2.9 \mu \mathrm{l}$ of $\mathrm{H}_{2} \mathrm{O}$ and $0.1 \mu \mathrm{l}$ of DNA polymerase (Taq DNA Polymerase, GenScript). PCR conditions were as follows: initial denaturation at $94{ }^{\circ} \mathrm{C}$ for 3 min followed by 35 cycles of denaturation at $94{ }^{\circ} \mathrm{C}$ for $15 \mathrm{~s}$, annealing at $60^{\circ} \mathrm{C}$ for $30 \mathrm{~s}$, elongation at $72^{\circ} \mathrm{C}$ for $45 \mathrm{~s}$ and a final elongation at $72^{\circ} \mathrm{C}$ for $7 \mathrm{~min}$. The PCR 
products were purified with Exonuclease I and shrimp alkaline phosphatase and sequenced using dye-terminator chemistry (kit v.3, ABI 3130XL) on an Applied Biosystems Prism 3700 DNA automated sequencer.

\section{Quantitative real-time PCR ( $q P C R$ )}

A quantitative real-time PCR (qPCR) was performed using a $\mathrm{ViiA}^{\mathrm{m}} 7$ real-time thermal cycler (Applied Biosystems) in the index patients and their parents to detect abnormalities in dosage in IFT140. An additional file contains a list of primer sequences used for qPCR (see Additional file 2). qPCR reactions were run in triplicate in a total volume of $12 \mu \mathrm{l}$ in each well and contained $6 \mu \mathrm{l}$ of SYBR Green PCR Master Mix (Applied Biosystems), $5 \mu \mathrm{l}$ of genomic DNA $(2 \mathrm{ng} / \mu \mathrm{l})$, and $0.5 \mu \mathrm{l}$ of forward and reverse primer each $(10 \mu \mathrm{mol} / \mathrm{l})$. The following program was applied: stage $1: 95^{\circ} \mathrm{C}$ for $10 \mathrm{~min}$; stage 2 : $95^{\circ} \mathrm{C}$ for $30 \mathrm{~s}, 60^{\circ} \mathrm{C}$ for $30 \mathrm{~s}, 72^{\circ} \mathrm{C}$ for $1 \mathrm{~min}$, for $40 \mathrm{cy}-$ cles and stage $3: 95^{\circ} \mathrm{C}$ for $15 \mathrm{~s}, 60^{\circ} \mathrm{C}$ for $1 \mathrm{~min}$ and $95^{\circ} \mathrm{C}$ for $15 \mathrm{~s}$. The results were normalized to albumin gene (ALB) and the copy number of each of the targeted IFT140 exons was determined by using a comparative DDCt method thereby using normal healthy control DNA as a calibrator. We performed sex determination of samples in reference to factor VIII gene (F8) located on $\mathrm{X}$ chromosome as a measure of quality assurance.

\section{Duplex PCR}

In order to confirm the presence of IFT140 tandem duplication, a duplex PCR was performed in both patients and their parents as described by Geoffroy et al. [12].

\section{Supplementary information}

Supplementary information accompanies this paper at https://doi.org/10. 1186/s13023-020-1303-2.

Additional file 1. List of genes and SNPs included in the NGS gene panel

Additional file 2. List of primer sequences used for PCR, Sanger sequencing and $\mathrm{qPCR}$.

\section{Abbreviations \\ CED: Cranioectodermal dysplasia; ESRD: End-stage renal disease; ExAC: Exome Aggregation Consortium; IFT: intraflagellar transport; NGS: next generation sequencing; TRP: tetratricopeptide repeats; WES: whole exome sequencing}

\section{Acknowledgements}

We thank the Sensenbrenner families for their participation and Professor Jonathan Eggenschwiler for editing the manuscript.

\section{Authors' contributions}

JWS: designed and supervised the study, wrote the manuscript; RP: performed clinical examinations, collected blood samples, wrote the clinical part of the manuscript; JWS, EO, AW: conducted molecular studies; EO: wrote the methods section of the manuscript; $\mathrm{HHA}, \mathrm{MO}, \mathrm{MS}$ : performed and analysed whole exome sequencing data; $\mathrm{HHA}, \mathrm{MO}, \mathrm{MS}, \mathrm{AJ}$ : critically revised the manuscript; EO, AW, AJ: performed data analysis; ALB: designed the study and was involved in clinical interpretation; $A W^{*}$ : contributed in the clinical examination and clinical data collection.* Anna Wasilewska. All authors read and approved the final manuscript.

\section{Funding}

This work was supported by the Poznan University of Medical Sciences (50214-01126186-10642) to JWS, grants from the Polish National Science Centre, Poland UMO-2016/23/N/NZ5/02577 to EO and UMO-2016/22/E/NZ5/00270 to AJ. MS acknowledges funding from the European research Council (ERC; ERC starting grant TREATCilia, grant no. 716344) and the German Research Foundation (Deutsche Forschungsgemeinschaft DFG, SFB1140, KIDGEM). $\mathrm{HHA}$ received support from the Netherlands Organization for Scientific Research (NWO Veni-91613008) and the Dutch Kidney Foundation (CP11.18 'KOUNCIL').

\section{Availability of data and materials}

All data relevant to the study are included in the article or uploaded as supplementary information.

\section{Ethics approval and consent to participate}

The study was approved by the Bioethics Committee at the Poznan University of Medical Sciences according to the clinical practice guidelines and the Polish law.

\section{Consent for publication}

Written informed consent for publication was obtained from the parents.

\section{Competing interests}

The authors declare that they have no competing interests.

\section{Author details}

${ }^{1}$ Department of Medical Genetics, Poznan University of Medical Sciences, Rokietnicka 8 Street, 60-608 Poznan, Poland. ²Department of Perinatology, Medical University of Bialystok, Bialystok, Poland. ${ }^{3}$ Department of Human Genetics, Radboud University Medical Center, Nijmegen, The Netherlands. ${ }^{4}$ Radboud Institute for Molecular Life Sciences, Radboud University Medical Centre, Nijmegen, The Netherlands. ${ }^{5}$ Center for Pediatrics and Adolescent Medicine, Freiburg University Hospital, Freiburg University Faculty of Medicine, Freiburg, Germany. ${ }^{6}$ Department of Pathology and Laboratory Medicine, Dalhousie University, Halifax, Nova Scotia, Canada. ${ }^{7}$ IWK Health Centre, Clinical Genomics Laboratory, Halifax, Nova Scotia, Canada. ${ }^{8}$ Department of Pediatrics and Nephrology, Medical University of Bialystok, Bialystok, Poland.

Received: 16 August 2019 Accepted: 13 January 2020

Published online: 01 February 2020

\section{References}

1. Arts H, Knoers N. Cranioectodermal Dysplasia. Seattle: University of Washington; 1993-2018.

2. Lin AE, Traum AZ, Sahai I, Keppler-Noreuil K, Kukolich MK, Adam MP, Westra $\mathrm{SJ}$, Arts HH. Sensenbrenner syndrome (Cranioectodermal dysplasia): clinical and molecular analyses of 39 patients including two new patients. Am J Med Genet Part A. 2013;161:2762-76.

3. Walczak-Sztulpa J, Eggenschwiler J, Osborn D, et al. Cranioectodermal dysplasia, Sensenbrenner syndrome, is a ciliopathy caused by mutations in the IFT122 gene. Am J Hum Genet. 2010;86:949-56.

4. Gilissen $\mathrm{C}$, Arts $\mathrm{HH}$, Hoischen A, et al. Exome sequencing identifies WDR35 variants involved in Sensenbrenner syndrome. Am J Hum Genet. 2010;87: 418-23.

5. Arts HH, Bongers EMHF, Mans DA, et al. C14ORF179 encoding IFT43 is mutated in Sensenbrenner syndrome. J Med Genet. 2011;48:390-5.

6. Bredrup C, Saunier S, Oud MM, et al. Ciliopathies with skeletal anomalies and renal insufficiency due to mutations in the IFT-A gene WDR19. Am J Hum Genet. 2011;89:634-43.

7. Girisha KM, Shukla A, Trujillano D, Bhavani GS, Hebbar M, Kadavigere R, Rolfs A. A homozygous nonsense variant in IFT52 is associated with a human skeletal ciliopathy. Clin Genet. 2016;90:536-9.

8. Bayat A, Kerr B, Douzgou S, DDD Study. The evolving craniofacial phenotype of a patient with Sensenbrenner syndrome caused by IFT140 compound heterozygous mutations. Clin Dysmorphol. 2017;26:247-51. 
9. Perrault I, Saunier S, Hanein S, et al. Mainzer-Saldino syndrome is a Ciliopathy caused by IFT140 mutations. Am J Hum Genet. 2012;90:864-70.

10. Oud MM, Latour BL, Bakey Z, et al. Cellular ciliary phenotyping indicates pathogenicity of novel variants in IFT140 and confirms a Mainzer-Saldino syndrome diagnosis. Cilia. 2018;7:1.

11. Forbes TA, Howden SE, Lawlor K, et al. Patient-iPSC-derived kidney Organoids show functional validation of a Ciliopathic renal phenotype and reveal underlying Pathogenetic mechanisms. Am J Hum Genet. 2018;102: 816-31.

12. Geoffroy V, Stoetzel C, Scheidecker S, et al. Whole-genome sequencing in patients with ciliopathies uncovers a novel recurrent tandem duplication in IFT140. Hum Mutat. 2018:39:983-92.

13. Schmidts M, Frank V, Eisenberger T, et al. Combined NGS approaches identify mutations in the Intraflagellar transport gene IFT140 in skeletal Ciliopathies with early progressive kidney disease. Hum Mutat. 2013;34:71424.

14. Peña-Padilla C, Marshall CR, Walker S, et al. Compound heterozygous mutations in the IFT140 gene cause Opitz trigonocephaly C syndrome in a patient with typical features of a ciliopathy. Clin Genet. 2017;91:640-6.

15. Schmidts M. Clinical genetics and pathobiology of ciliary chondrodysplasias. J Pediatr Genet. 2014;3:46-94.

16. Richards S, Aziz N, Bale S, et al. Standards and guidelines for the interpretation of sequence variants: a joint consensus recommendation of the American College of Medical Genetics and Genomics and the Association for Molecular Pathology. Genet Med. 2015;17:405-23.

17. Renkema KY, Giles RH, Lilien MR, Beales PL, Roepman R, Oud MM, Arts HH, Knoers NVAM. The KOUNCIL consortium: from genetic defects to therapeutic development for Nephronophthisis. Front Pediatr. 2018;6:131.

18. Molinari E, Sayer JA. Emerging treatments and personalised medicine for ciliopathies associated with cystic kidney disease. Expert Opin Orphan Drugs. 2017;5:785-98.

19. Piperno G, Siuda E, Henderson S, Segil M, Vaananen H, Sassaroli M. Distinct mutants of retrograde intraflagellar transport (IFT) share similar morphological and molecular defects. J Cell Biol. 1998;143:1591-601.

20. Neugarten J, Golestaneh L. Gender and the prevalence and progression of renal disease. Adv Chronic Kidney Dis. 2013;20:390-5.

21. Zaffanello M, Diomedi-Camassei F, Melzi ML, Torre G, Callea F, Emma F. Sensenbrenner syndrome: a new member of the hepatorenal fibrocystic family. Am J Med Genet Part A. 2006;140A:2336-40.

22. Moosa S, Obregon MG, Altmüller J, Thiele H, Nürnberg P, Fano V, Wollnik B. Novel IFT122 mutations in three Argentinian patients with cranioectodermal dysplasia: expanding the mutational spectrum. Am J Med Genet Part A. 2016;170:1295-301.

23. Walczak-Sztulpa J, Wawrocka A, Sobierajewicz A, et al. Intrafamilia phenotypic variability in a Polish family with Sensenbrenner syndrome and biallelic WDR35mutations. Am J Med Genet A. 2017:173:1364-8.

24. Lelieveld SH, Reijnders MRF, Pfundt R, et al. Meta-analysis of 2,104 trios provides support for 10 new genes for intellectual disability. Nat Neurosci. 2016;19:1194-6.

\section{Publisher's Note}

Springer Nature remains neutral with regard to jurisdictional claims in published maps and institutional affiliations.

Ready to submit your research? Choose BMC and benefit from:

- fast, convenient online submission

- thorough peer review by experienced researchers in your field

- rapid publication on acceptance

- support for research data, including large and complex data types

- gold Open Access which fosters wider collaboration and increased citations

- maximum visibility for your research: over $100 \mathrm{M}$ website views per year

At $\mathrm{BMC}$, research is always in progress.

Learn more biomedcentral.com/submissions 